\title{
A DEPRESSED COLLECTOR SYSTEM FOR A QUASI-OPTICAL GYROTRON WITH PRECISELY CONTROLLED MAGNETIC FLUX LINES*1
}

\author{
A Singh, ${ }^{3}$ V.L. Granatstein, ${ }^{3}$ G. Hazel, ${ }^{3}$ \\ G. Saraph, ${ }^{3}$ J.M. Cooperstein, ${ }^{* * 2.3}$ \\ and T. Hargreaves ${ }^{4}$ \\ ${ }^{3}$ Laboratory for Plasma Research \\ University of Maryland \\ College Park, Maryland 20742 \\ ${ }^{4}$ Naval Research Laboratory \\ Washington, $D C$
}

Received February 12, 1991

\begin{abstract}
Design of a depressed collector system for a quasi-optical gyrotron, which had a severe constraint on the maximum allowable radius of the collector region is outlined. The needs for unwinding of spent beam and for energy sorting could be accommodated by precise control of the magnetic field profile, especially in the collector region. Techniques used for defining and obtaining such profiles; and for dovetailing the profile with the collector geometry are discussed. Results on profiles and electron trajectories are presented, which demonstrate the feasibility of the design. From primary electron trajectories a collector efficiency of up to $68 \%$ has been calculated for a three collector design.
\end{abstract}

\section{INTRODUCTION}

Some of the main issues involved in design of energy recovery systems for gyrotrons are: a) separation of the paths of the spent beam and the rf output, b) unwinding of the spent beam, and c) energy sorting before collection at different depressed potentials. As regards the first item, quasi-optical gyrotrons (QOG) have the advantage that the two paths are naturally separated.

\footnotetext{
${ }^{1 *}$ This work is supported by the U.S. Department of Energy.

${ }^{2 * *}$ Permanent address: University of Michigan, Ann Arbor, MI
} 
The spent beam emerging from the rf interaction region still has a large portion of its energy in transverse motion. That needs to be converted into axial motion, as far as possible. With that accomplished the beam energy can be recovered by an electrostatic retarding field., which is largely in the axial direction. In large-orbit gyrotrons this unwinding of the beam can be done by a non-adiabatic transition through a magnetic cusp[1]. In the case of small orbit gyrotrons, initial unwinding can be done by adiabatic expansion of the beam, followed by a non-adiabatic transition[2]. During adiabatic expansion, the radius of the beam increases in the ratio of $B_{z}^{-1 / 2}$, while $\alpha$, the ratio of $v_{\perp}$ to $v_{\|}$, decreases according to the relation

$$
\alpha=\left[\left(\alpha_{0}^{-2}+1\right) f_{m}-1\right]^{-1 / 2}
$$

where $\alpha_{0}$ corresponds to the starting value of $\alpha$, and $f_{m}$ is the ratio of the starting value of $B_{z}$ to its value after beam expansion. For the case of millimeter wave gyrotrons operating in the fundamental modes, the value of $B_{z}$ in the rf interaction region is several Tesla. The beam radius after adiabatic expansion can thus get expanded to unmanageably large values, if the unwinding of the beam is done by adiabatic expansion all the way.

To restrict the radius of the beam in the collector region, the adiabatic expansion can be followed by a non-adiabatic transition. This leads to beam heating which can be minimized by suitable choice of profile of the non-adiabatic transition[3]. A transition which is neither adiabatic nor purely non-adiabatic can be used while limiting the radius of the beam, as well as the beam heating[4]. This requires precise tailoring of the magnetic field profile.

After rf interaction the spent beam has a spectrum of energies. For optimum energy recovery the beamlets of different energy need to be collected at different depressed potentials. This energy sorting can be done by the electrostatic field if the magnetic field has been reduced to a low enough value in the collector region: such that the force exerted by the electrostatic field dominates the force due to magnetic field. This is not always possible, due to limitations on the radius of the collectors. However, energy sorting can also be done by 
non-adiabatic transitions in the magnetic field. This is because the slower electrons will more closely follow rapid changes in the direction of lines of magnetic flux than their more energetic counterparts. Ultimately it is a combination of all these techniques of unwinding the beam, and of energy sorting that leads to best results in a given situation.

In the present study the objective was to examine the feasibility of a design of multiple depressed collectors (MDCs) for the quasioptical gyrotron (QOG) under development at the Naval Research Laboratory (NRL) [5]. Encouraging results have been obtained by using a single depressed collector[6]. A collector efficiency of the order of $50 \%$ could be measured. However, this corresponded with operation parameters where the overall efficiency came to $12.5 \%$ and the electronic efficiency was relatively low. This is understandable since with a low value of electronic efficiency the spread in the energy of the beam is small. Thus a single depressed collector can be adequate for that case. The best overall efficiency was $16.1 \%$ with a collector efficiency of $34 \%$. However, for high overall efficiency both the electronic and the collector efficiency have to be high. In that case it is necessary to go to a design with multiple depressed collectors.

The maximum radius allowable with the existing experimental set up was less than $18 \mathrm{~cm}$ because of a liquid helium feed tube at that radius (Fig. 1). This restricts the extent of adiabatic expansion that can be used and correspondingly restricts the adequacy of unwinding of the beam, before it enters the collector region. It was thus necessary to consider an approach which involves tailoring of the magnetic field profile, and dovetailing it with the geometry of the collectors. An adiabatic profile is followed by a field configuration controlled over the full range of $r$ and $z$ in the collector region. This control is exercised by use of additional coils and pole piece. A schematic diagram showing the relationship of the QOG to an early example of the design of multiple depressed collectors with additional coils is shown in Fig. 2.

The techniques and computer code that have been developed for precise tailoring of magnetic field profile could be applied elsewhere 


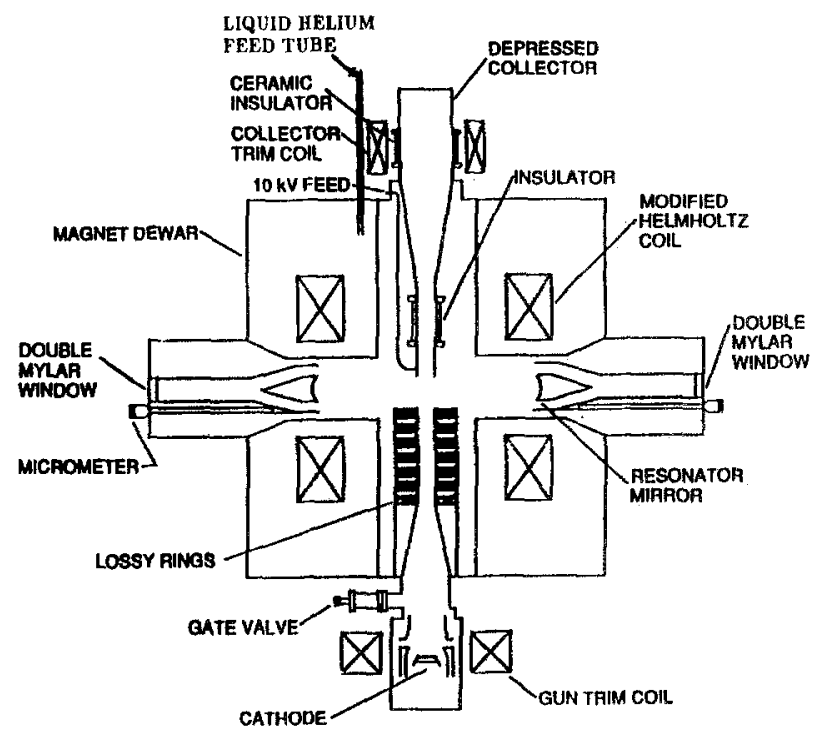

Figure 1: Schematic diagram of quasioptical gyrotron (QOG) (courtesy NRL).

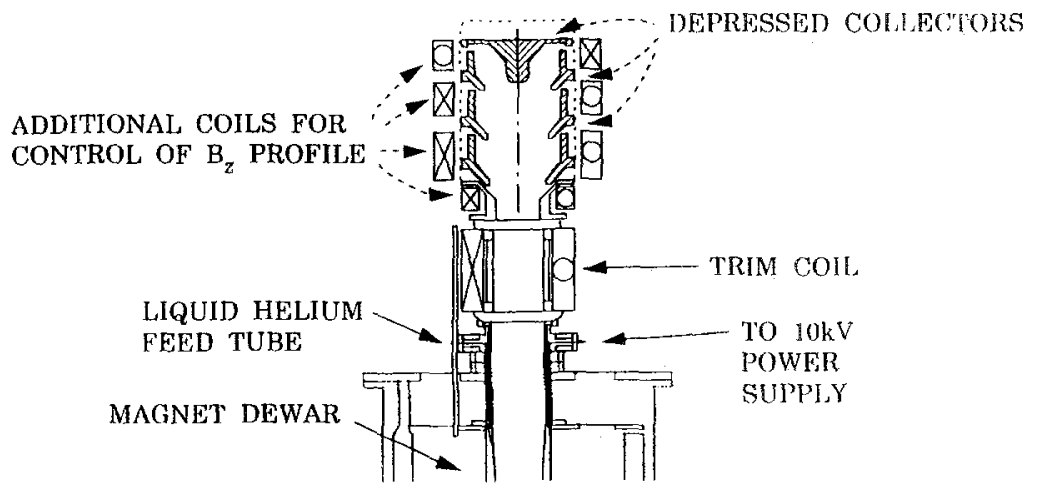

Figure 2: Schematic diagram showing the relationship of the QOG to an early version of multiple-depressed collector design, along with additional coils for control of magnetic field profile. 
also. Section 2 describes the code and the approach used. Section 3 presents the manner in which electron trajectories were simulated by numerical computation. In Section 4 some stages of design optimization are presented. Conclusions are discussed in Section 5 .

\section{COMPUTER CODE FOR PRECISE TAILORING OF MAGNETIC FLUX LINES}

We have developed a code that allows us to perform the following functions:

i) Outlines of the geometry and locations of pole pieces, coils and geometry of the collectors can be superimposed on the video screen, along with contour plots of constant values of $r a_{\theta}$, or of $B_{r}$, or $B_{z}$ (where $a_{\theta}$ is the azimuthal component of the magnetic vector potential).

ii) The field distributions due to unit current in individual coils are separately evaluated using POISSON and are stored as files in the memory of the computer. In this as well as the previous step only the essential data is retained so as not to overload the available memory. For instance, the differential coefficients of Bfield components given in the output of POISSON are deleted. So too the minute steps used in delineating the geometry of the collector for purposes of electron trajectory simulation are substituted by an algorithm for a relatively coarse drawing of lines between the main turning points in the boundary. Such a geometry is adequate for the purpose of seeing the relationship of magnetic flux lines to the collector electrodes. In this manner a personal computer can be used for on-line design, as explained in the next paragraphs.

iii) The values of the current in different coils can be adjusted online, and their combined effect on the three kinds of contours mentioned above, can be ascertained vis-a-viz electrode geometry and the desired contours obtained. Contours for the electrodes, the coils, the pole pieces, $B_{z}, B_{r}, a_{\theta}$ are shown in different colors for convenience. One to eight windows can be shown on the video screen. Text showing other parameters is also on 
display. The desired values $B_{z}, B_{r}$ and $a_{\theta}$ to be used as parameters for contours or profiles to be displayed can be specified. The geometry can be displayed in the same proportion for both axes, or with proportion adjusted so as to fill up the window. The current through each coil or group of coils can be modified incrementally in a very simple way. The increment/decrement step can be adjusted to become finer as the design proceeds. At successive stages of adjustment of currents the previous contours or profiles can be either cleared or retained for purposes of comparison. The value of the radius at which the $B_{z}$ and $B_{r}$ profiles an given can also be specified to correspond to the value of maximum interest (and not just the axis). Precise locations and exact values of magnetic field parameters can be read out digitally, using a 'mouse'. An early example of such contours of $r a_{\theta}, B_{z}$ and the collector electrodes is shown in Fig. 3. This shows a geometry of the collectors which was later optimized as discussed later in this section and in section 4 .

iv) For the actual field configuration POISSON is re-run with the current values obtained in step iii). This takes into account any non-linear effects in the permeability of shield material.

These techniques allowed us to obtain precise values of current for given coils for the desired portrait of flux lines. The constraint on the radius of the collector gives very limited room for beamlets of different energies to spread out. But by configuring the electrode surfaces to lie nearly parallel to the lines of constant $r a_{\theta}$ it was possible to make use of relatively small variations in angle of trajectories emerging from a non-adiabatic transition to get energy sorting.

\section{SIMULATION OF ELECTRON TRAJECTORIES}

The choice of the energy spectrum of the spent beam was based on rf simulations done at NRL as shown in Fig. 4. The energy spectrum was divided into five groups centered at 40,60,80,100, and $120 \mathrm{kV}$. The value of $\alpha$ was calculated for each group, assuming that the rf interaction process affected the energy of transverse motion, and not that of longitudinal motion. This value was transformed to a reduced 


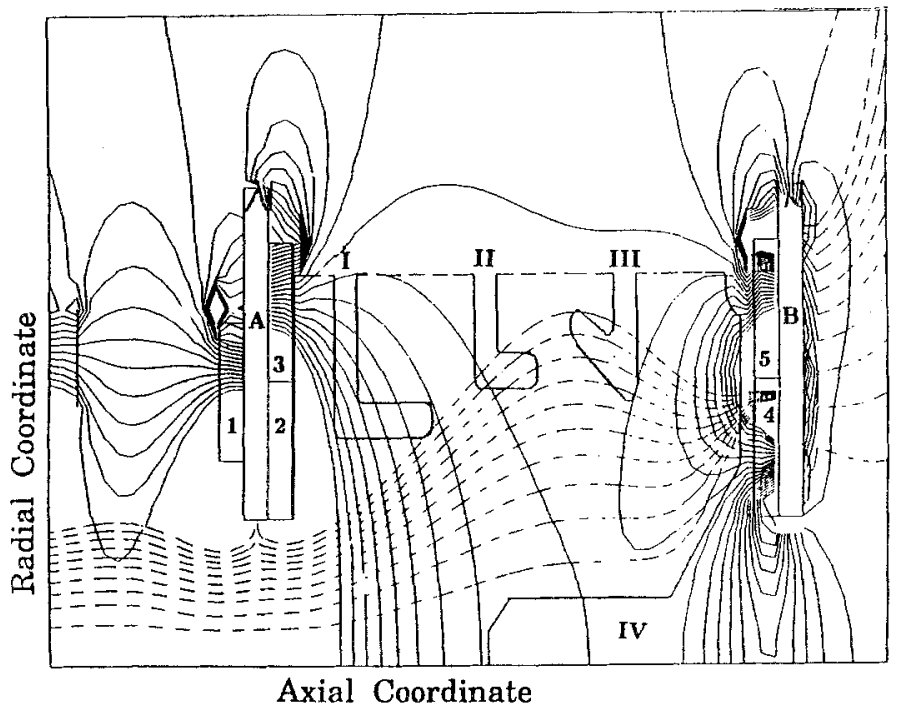

Figure 3: An example of combined portrait of collectors (I to IV), pole pieces (A, B), and coils ( 1 to 5 ). Flux lines are shown as dotted lines, contours of constant $B_{z}$ are shown as solid lines.

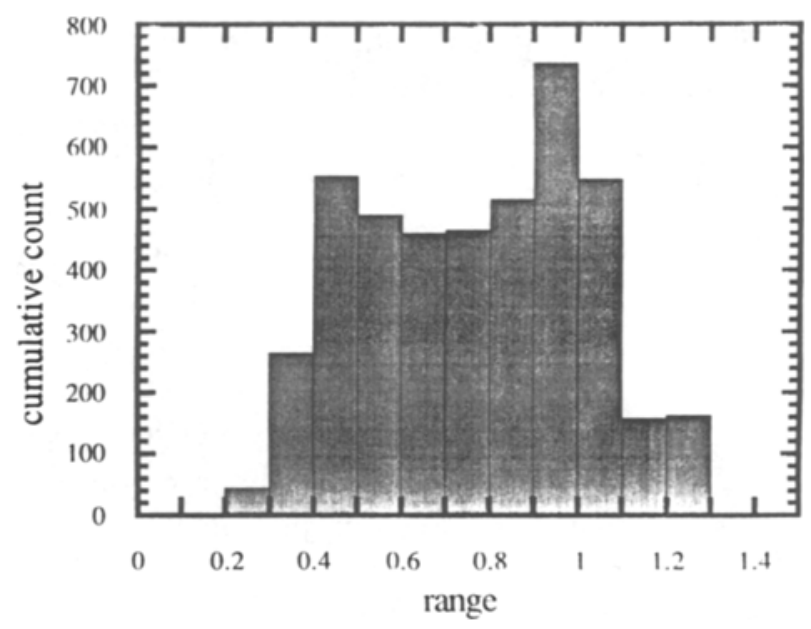

Figure 4: The energy spectrum of the spent beam, based on a simulation done at NRL. 
value of $\alpha$ due to adiabatic transition up to a point where the magnetic field had fallen from $50 \mathrm{kG}$ to $5 \mathrm{kG}$ as per Eq. (1). The radius of the beam was correspondingly increased in the ratio of $B^{-1 / 2}$. Simulation of electron trajectories in the region beyond the point where the $B_{z}$ field component had fallen to $5 \mathrm{kG}$, was done by using the code EGUN[7]. In this code the values of alpha and alpha (transverse) depend upon the phase of the gyration at a given moment. In order to get four representative phases, the magnitude of $\alpha$ obtained from Eq. (1) was used with positive and negative sign keeping alpha (trans) as zero; and then vice versa. This gave four rays for each energy value, making a total of 20 rays. The vector potential array, and the geometry of the electrodes interactively designed as described in section 2 were also inserted into the code EGUN as input parameters.

In order to estimate the collector efficiency, a computer program has been written which extracts data from one of the output files of the code EGUN, "htraj", regarding the current carried by each ray as well as its energy at the entrance to the collector region and at the end of its trajectory. The two summations of the products of current and energy values give the incoming energy and the energy dissipated on the collectors; the difference between the two being the energy recovered. The calculated collector efficiency is printed out along with the summary data on the rays.

\section{DESIGN OPTIMIZATION}

Simulations of trajectories in configurations shown in Figs. 2 and 3 , gave collector efficiencies estimated as $49 \%$ and $55 \%$. The limiting factors were a) the tendency of some of the rays to turn around before getting collected, and b) inadequate energy sorting. In some cases the ray that was reflected back turned around again as it approached the region of high $B_{z}$ field. This was particularly true where a peak in $B_{z}$ was tried as a non-adiabatic transition just before the collector region. It was thus necessary to further refine the magnetic field profile and the electrode geometry.

Using the techniques outlined in section 2, a design was evolved which is shown in Fig. 5. The magnetic circuit augments the existing 
set of superconducting coils, and a trim coil. An iron plate is added to act as a shield between the transition region and the collector region. Besides this there is one pancake shaped coil on the upstream side of the shield which controls the entrance flux. On the collector side there are two pairs of pancake shaped coils with different radii at the beginning and the end of the collector region. Independent control of current in these five coils, together with choice of current in the trim coil is used as data input to the code described above.

The magnetic flux lines are made to curve upwards near the entrance. Electron trajectories corresponding to this configuration are shown in Fig. 6. The two lowest energy groups of 40 to $60 \mathrm{keV}$ follow the flux lines more closely and get collected at the first depressed collector. The higher energy groups proceed to collector number two. The flux lines are bent again towards the axis in the region of collector number two. This helps to restrict the maximum radius of the collector. Furthermore, this second bending provides further energy sorting, so that the middle energy groups land on collector number three. Because of the restriction on dimensions the energy sorting is not perfect. However, there are no reflections of any particle in these energy groups. Also the energy is deposited rather evenly on the collector surfaces. With optimum potentials on the collectors, an estimated efficiency of $68 \%$ could be obtained on the basis of the primary particle trajectories. This figure may be revised upwards by further optimization and downwards by a few percentage points by including the effect of backscattered electrons. The optimization would include smoothing the profile of the magnetic field in the transition region. Presently this profile has peaks and troughs because of the existing axial geometry. The trim coil is placed at a distance from the interaction cavity determined by the height of the Dewar. This implies that $B_{z}$ falls to a relatively low value, and has to be raised by the trim coil to prevent the beam from hitting the walls of the transition region. This leads to a trough and a peak. In a new design or by adding additional coils the field profile can be smoothened. In this way the energy sorting could be effected by a new adiabatic transition at essentially the entrance to the collector only. Presently the troughs and peaks of the field also cause some mixing of beamlets 


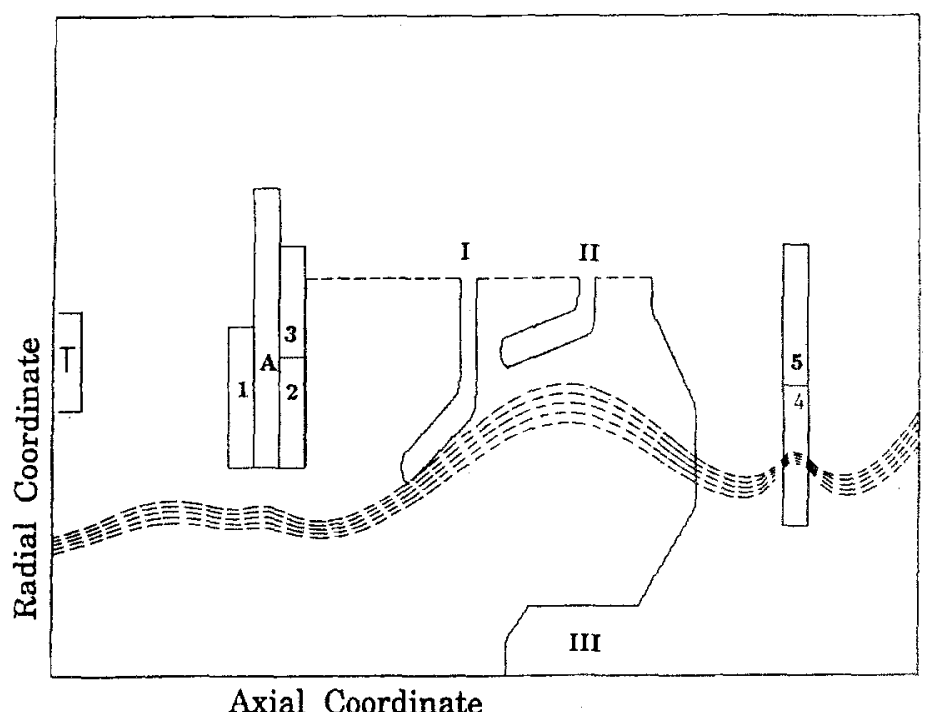

Figure 5: Modified geometry and some optimized lines of magnetic flux.

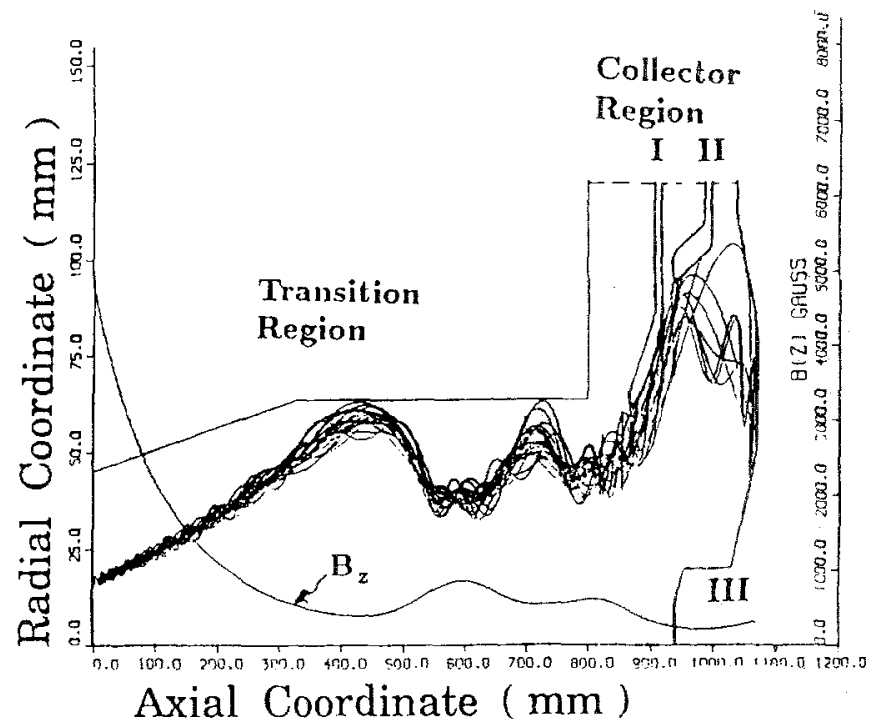

Figure 6: Electron trajectories in the transition region and the collector region. (Note expanded scale for $r$ compared to $z$ ). 
of different energies vis-a-viz the energy sorting caused by a single non-adiabatic transition at the entrance to the collector. We note that even a $63 \%$ collector efficiency can give an overall efficiency of $36 \%$ for an electronic efficiency of $17 \%$. The collector efficiency can go higher for a smaller electronic efficiency which corresponds to a smaller energy spread, and vice versa.

\section{CONCLUSIONS}

Approaches to the configuration of a magnetic field and a computer code for synthesizing it have been developed. The code also makes it possible to tailor the collector field geometry in an interactive mode. For the case of a quasi-optical gyrotron the feasibility of reaching a collector efficiency in the mid-sixties is demonstrated while accommodating a severe restriction in the maximum allowable radius.

\section{REFERENCES}

1. A. Singh, W. Lawson, D. Goutos, W.R. Hix, C.D. Striffler, V.L. Granatstein, and W.W. Destler, "Beam conditioning for electron energy recovery systems in devices employing axisencircling beams," Int. J. Electronics 65, pp. 351-368 (1988).

2. M.E. Read, A.J. Dudas, W. Lawson, and A. Singh, "Depressed collectors for high power gyrotrons," IEEE Trans. on Electron Devices 17, 1579-1589 (1990).

3. A. Singh, V.L. Granatstein, C. Casey and W.W. Destler, "Non adiabatic transition of beams in gyrotrons through various magnetic field profiles," to be published in the Int. J. of Electronics.

4. A. Singh, G. Hazel, V.L. Granatstein and G. Saraph, "Efficiency enhancement of small-orbit gyrotrons by energy recovery from spent beam," sent for publication to the Int. J. of Electronics.

5. A.W. Fliflet, T.A. Hargreaves, W.M. Wallace, R.P. Fischer, and M.L. Barsanti, "Initial operation of a high-power quasi-optical gyrotron," IEEE Trans. on Plasma Science 18, 306-312 (1990). 
6. T.A. Hargreaves, A.W. Fliflet, R.P. Fischer and M.L. Barsanti, "Depressed collector performance on the NRL quasioptical gyrotron," Conference Digest 15th Int. Conf. on Infrared and Millimeter Waves, Orlando, FL, Dec. 1990, pp. 330-332.

7. W.B. Herrmannsfeldt, "EGUN - An electron optics and gun design program," Stanford Linear Accelerator Center, Report 331 , Oct. 1988. 